\title{
Targeted treatment for advanced soft tissue sarcoma: profile of pazopanib
}

This article was published in the following Dove Press journal:

OncoTargets and Therapy

15 March 2013

Number of times this article has been viewed

\author{
Rajeev Rajendra \\ Robin L Jones \\ Seth M Pollack \\ University of Washington/Fred \\ Hutchinson Cancer Research Center, \\ Seattle, WA, USA
}

\begin{abstract}
Soft tissue sarcomas comprise approximately $1 \%$ of all adult solid malignancies. While chemotherapy is the mainstay of treatment for patients with metastatic or inoperable disease, overall survival for these patients is approximately 12 months, highlighting the need for novel agents. Both laboratory and clinical data have suggested that antiangiogenic agents may have a role in the treatment of soft tissue sarcomas. Pazopanib is a multitargeted receptor tyrosine kinase inhibitor with antiangiogenic activity. The randomized, double-blind, placebo-controlled, Phase III PALETTE (pazopanib for metastatic soft-tissue sarcoma) study demonstrated improved progression-free survival in patients receiving pazopanib compared with placebo. In this review, we discuss the rationale and clinical evidence for the use of pazopanib in the treatment of metastatic and inoperable soft tissue sarcomas.
\end{abstract}

Keywords: pazopanib, metastatic, sarcoma

\section{Introduction}

Soft tissue sarcomas (STS) are a rare and heterogeneous group of mesenchymal tumors, comprising over 50 different histological subtypes and accounting for approximately $1 \%$ of adult solid malignancies. Individual histological subtypes show great differences in terms of genetic alterations, pathogenesis, and clinical behavior, varying from relatively indolent to highly aggressive disease. The median overall survival for patients with metastatic disease is approximately 12 months. ${ }^{1}$ Chemotherapy is the mainstay of treatment for patients with metastatic or inoperable disease. Schedules including doxorubicin or ifosfamide achieve objective responses in $15 \%-35 \%$ of patients. ${ }^{1}$ The last decade has seen the emergence of several novel agents with activity against certain STS entities. The introduction of imatinib and other tyrosine kinase inhibitors has revolutionized the treatment of gastrointestinal stromal tumors and has been a paradigm for targeted therapy in solid tumors. Paclitaxel has shown efficacy and good tolerability in angiosarcoma in a single-arm trial. ${ }^{2}$ Trabectedin was approved in Europe in 2007 for patients with STS, and has demonstrated considerable activity in leiomyosarcoma and myxoid/round cell liposarcoma. ${ }^{3}$ Gemcitabine with dacarbazine or docetaxel has demonstrated activity in uterine leiomyosarcoma. ${ }^{4,5}$ Despite these advances, the poor median progression-free survival of approximately 3 months and the median overall survival of approximately 12 months for all STS have remained unchanged. Given such poor outcomes, there is clearly an unmet need for novel therapeutic options in the arsenal for STS.

Several studies have suggested that angiogenesis plays an important role in the growth and spread of STS. Yoon et $\mathrm{al}^{6}$ demonstrated 10 -fold higher levels of vascular
Correspondence: Seth M Pollack

University of Washington/Fred

Hutchinson Cancer Research Center

D3-100, I 100 Fairview Avenue, Seattle,

WA, USA 98109

Tel + I 2066676629

Fax + I 2066677983

Email spollack@fhcrc.org 
endothelial growth factor (VEGF) by angiogenic profiling of STS compared with normal tissue controls. Indeed, high VEGF expression is an independent and prognostic indicator for increased risk of metastases and decreased overall survival. ${ }^{7}$ Further, blood VEGF levels in patients with STS are elevated compared with controls and are related to the histologic grade of the primary tumor. ${ }^{6,8-10}$ Increasing evidence has demonstrated that platelet-derived growth factor (PDGF) receptor signaling is also involved in tumor angiogenesis. ${ }^{11}$ Specifically, signaling through PDGF receptor- $\beta$ seems to play a critical role in the recruitment of the tumor pericytes responsible for development of functional capillaries and production of VEGF by the tumor stroma. ${ }^{11,12}$ There is some evidence that simultaneously targeting the VEGF and PDGF pathways may be more effective than targeting either pathway alone, leading some investigators to explore multitargeted receptor tyrosine kinase inhibitors. ${ }^{13,14}$

\section{Antiangiogenic agents in soft tissue sarcoma}

Sunitinib malate is an oral multitargeted tyrosine kinase inhibitor of VEGF receptors 1, 2, and 3, PDGF receptor- $\alpha$ and receptor- $\beta$, KIT, FMS-related tyrosine kinase 3 , RET, and the colony stimulating factor-1 receptor tyrosine kinases. In a multicenter Phase II trial of sunitinib for the treatment of nongastrointestinal stromal tumors, which included four patients with synovial sarcoma, 11 of 48 patients demonstrated stable disease, including one patient with synovial sarcoma who had stable disease following 16 weeks of therapy. ${ }^{15}$ A Phase II study of sorafenib, an inhibitor of Raf-1 in addition to VEGF receptor 2 and 3, PDGF receptor- $\beta$, FMS-related tyrosine kinase 3, and KIT in patients with metastatic or recurrent sarcomas demonstrated some activity in a group of 12 patients with synovial sarcoma, with a Kaplan-Meier estimate for progression-free survival of $42 \%$ at 3 months. ${ }^{16}$

A Phase I study of cediranib, a potent inhibitor of VEGF receptors 1,2 , and 3, and c-KIT in children and adolescents with refractory solid tumors, found objective responses in a sarcoma group, which included two patients with synovial sarcoma. One of the patients with synovial sarcoma in this study had a $67 \%$ reduction in tumor dimensions. ${ }^{17}$ Cediranib has shown promise in alveolar soft part sarcoma, a rare chemoresistant translocation-related sarcoma.

Brivanib, an oral dual inhibitor of VEGF receptors 1, 2, and 3 and fibroblast growth factor receptors 1, 2, and 3, was evaluated for the treatment of STS in a randomized Phase II discontinuation trial. Of the 251 patients with STS enrolled, there were seven partial responses, with an overall response rate of $2.8 \%$ and a disease control (overall response rate + stable disease) rate of $30 \%$. Median progression-free survival was 2.8 months from the initial 12-week lead-in period in a subgroup of high expressers of fibroblast growth factor receiving brivanib compared with 1.4 months for placebo controls $(P=0.08) .{ }^{18}$ These laboratory and clinical data suggest that antiangiogenic agents may have a role in the treatment of STS.

\section{Pazopanib \\ Preclinical data}

In the wake of the success of anti-VEGF drugs such as bevacizumab, GlaxoSmith Kline sought to target the VEGF and PDGF receptor kinases simultaneously. An initial screening of GlaxoSmithKline compounds identified two molecules causing potent inhibition of VEGF receptor 2 , ie, a quinazoline and a pyrimidine. Although crystallography for VEGF receptor 2 was not available at the time of the study, binding models were developed using the homologous fibroblast growth factor receptor and identified the quinazoline and pyrimidine regions as being responsible for the activity. This led to the development of 13 successive pyrimidine compounds with various and improved degrees of VEGF receptor binding, designed with the goal of good oral bioavailability. Pyrimidine 13, 5-[4-[(2,3-dimethyl-2H-indazol-6-yl)methylamino]-2pyrimidinyl]amino]-2-methyl-benzenesulfonamide, showed good potency against all VEGF receptors as well as PDGF receptor- $\beta$, c-KIT, fibroblast growth factor receptor 1 , and c-FMS in vitro, and was developed further to become the compound now known as pazopanib (GW786034). Further in vivo assays, including the mouse matrigel plug assay and the corneal micropocket assay, demonstrated potent inhibition of angiogenesis. The compound was able to inhibit xenografts from multiple solid tumors, and good murine bioavailability was observed. ${ }^{19}$

In mice given an intravenous bolus of VEGF, pazopanib blocked phosphorylation of VEGF receptor 2 compared with untreated controls. When the mice had previously received a single dose of pazopanib, inhibition of VEGF receptor 2 phosphorylation in the lungs was dose-dependent and timedependent. A single oral dose of pazopanib $30 \mathrm{mg} / \mathrm{kg}$ inhibited phosphorylation for more than 8 hours. These results suggest that optimal inhibition of VEGF receptor 2 phosphorylation requires plasma concentrations above $40 \mu \mathrm{mol} / \mathrm{L} \cdot{ }^{20}$ Further studies demonstrated that although efficacy was highly dose-dependent, adequate dosing could be safely achieved and that efficacy was strongly correlated with inhibition of the VEGF receptor. ${ }^{21}$ 


\section{Phase I data}

A Phase I clinical trial by Hurwitz et al assessed the safety, pharmacokinetics, and clinical activity of pazopanib in patients with refractory advanced-stage solid tumors. ${ }^{22}$ Patients were enrolled into sequential dose-escalating cohorts (50 and $100 \mathrm{mg}$ three times a week, 50-2000 mg once a day, and 300 and $400 \mathrm{mg}$ twice a day). Escalation or de-escalation was based on toxicities observed in the preceding dose cohort. Clinical response was assessed every 9 weeks. Sixty-three patients were treated (dose escalation, $n=43$; dose expansion, $\mathrm{n}=20$ ). Although a maximum tolerated dose was not achieved, absorption at doses $>800 \mathrm{mg}$ once daily were limited because of the oral bioavailability of pazopanib. Therefore, the $800 \mathrm{mg}$ per day dose cohort were selected for use in the Phase II and III clinical trials.

A plateau in steady-state exposure was observed at doses $\geq 800 \mathrm{mg}$ once daily, but it is still recommended that pazopanib be administered in between meals (either one hour before or two hours after food), because its absorption may be increased when administered with food. The mean elimination half-life at the $800 \mathrm{mg}$ dose was 31.1 hours. A mean target trough concentration $\left(\mathrm{C}_{24}\right) \geq 15 \mu \mathrm{g} / \mathrm{mL}(34 \mu \mathrm{mol} / \mathrm{L})$ was achieved at $800 \mathrm{mg}$ once daily. Three patients had partial responses, with stable disease of $\geq 6$ months observed in 14 patients, and clinical benefit was generally observed in patients who received doses $\geq 800 \mathrm{mg}$ once daily or $300 \mathrm{mg}$ twice daily. Hypertension, diarrhea, hair depigmentation, and nausea were the most frequent drug-related adverse events, the majority of which were grade 1 or 2 . Hypertension was the most frequent grade 3 adverse event. Four patients experienced dose-limiting toxicities at $50 \mathrm{mg}, 800 \mathrm{mg}$, and $2000 \mathrm{mg}$ once daily. This study demonstrated that pazopanib was generally well tolerated and showed antitumor activity across various tumor types, with a dose of $800 \mathrm{mg}$ once daily selected for Phase II studies. ${ }^{22}$

The pharmacokinetic characteristics of pazopanib are similar to those of sunitinib and sorafenib. Like other tyrosine kinase inhibitors, pazopanib is highly proteinbound. Furthermore, pazopanib, sunitinib, and sorafenib are all metabolized in the liver and eliminated via the feces. However, pazopanib undergoes significantly less renal elimination than sorafenib and sunitinib. ${ }^{23}$ Pazopanib is primarily metabolized by cytochrome $\mathrm{P} 450$ (CYP)3A4, but interactions with CYP3A4 inducers or inhibitors are not well characterized. ${ }^{22}$ Coadministration of strong CYP3A4 inhibitors (eg, clarithromycin or ketoconazole), grapefruit juice, and CYP3A4 inducers (eg, rifampin) should be avoided when possible.

\section{Phase II data}

A single-arm Phase II trial (European Organisation for the Research and Treatment of Cancer study 62034) treated 142 patients with intermediate-grade or high-grade advanced STS. The study included patients who were ineligible for chemotherapy or had received no more than two prior cytotoxic agents for advanced disease. Pazopanib $800 \mathrm{mg}$ was given daily. The primary end point was progressionfree survival at 12 weeks. Secondary end points were response, safety, and overall survival. Four different strata were studied, ie, adipocytic STS, leiomyosarcoma, synovial sarcoma, and other STS types. The adipocytic STS stratum was closed after analysis showed insufficient activity, with progression-free survival at 12 weeks of only $26 \%$. The remaining cohorts demonstrated progression-free survival at 12 weeks of $44 \%$ in the leiomyosarcoma group, $49 \%$ in the synovial sarcoma stratum, and 39\% in the stratum consisting of other STS types. Compared with historical controls treated with second-line chemotherapy, progression-free and overall survival were prolonged in the three cohorts in whom the primary end point was reached. The most frequent drug-related toxicities were hypertension, fatigue, hypopigmentation, and nausea. Other toxicities included liver enzyme elevations, myelosuppression, and proteinuria, most of which were grades 1 to 2 . The most frequent grades 3 to 4 toxicities were hyperbilirubinemia (6.3\%), hypertension (7.7\%), and fatigue $(7.7 \%) .^{24}$

\section{Phase III data}

These promising findings led to the multicenter, international, double-blind, placebo-controlled Phase III PALETTE (European Organisation for the Research and Treatment of Cancer study 62072) trial. ${ }^{25}$ PALETTE compared pazopanib and placebo, with 2:1 randomization of participants (and no crossover) who had received at least two lines of chemotherapy for metastatic nonadipocytic STS. Patients $(n=369)$ were randomly assigned to receive either pazopanib $800 \mathrm{mg}$ once daily $(n=246)$ or placebo $(n=123)$ until disease progression or development of unacceptable toxicity. The median age of the participants was 56 years, and median duration of follow-up was 15 months. The primary end point was progression-free survival, assessed by independent central review. The progression-free survival in patients receiving pazopanib was superior to that in those receiving placebo, with a median of 4.6 months and 1.6 months, respectively (hazard ratio $=0.31 ; P<0.0001$ ). In additional, Cox models revealed a significant benefit for progression-free survival across all histological subtypes. 
A trend towards improved overall survival was also seen in the pazopanib arm (12.5 months versus 10.7 months; hazard ratio $0.86, P=0.25$ ), but this was not statistically significant. After a 15-month follow-up analysis, the median overall survival of 10.7 months in the placebo group was approximately 3 months longer than that estimated in the trial design. Further, the study was essentially underpowered to detect the proposed 3-month overall survival benefit for pazopanib. The authors suggested that the unexpectedly long overall survival time observed in the placebo group might be due to the administration of post-progression therapy, which was not recorded. Whether patients in the control arm received antiangiogenic agents after progression in subsequent trials is also unclear, but might have influenced overall survival. Another possible reason for the lack of overall survival benefit was the occurrence of a "rebound" effect that accelerated progression after patients stopped pazopanib therapy. Theoretical causes of such a rebound effect include increased circulating levels of angiogenic peptides or increased invasion and metastasis. ${ }^{26}$

It is noteworthy that the patients included in this study had very poor prognoses, as shown by the low median progression-free survival and high frequency of adverse events in the placebo group. The most common grade 3 or 4 adverse events included hypertension (14\%) and fatigue (13\%), and other events of special interest included myocardial dysfunction $(2 \%)$, venous thromboembolism $(3 \%)$, and pneumothorax $(0.4 \%) .{ }^{27}$

\section{Limitations of PALETTE study}

The PALETTE study was the first randomized Phase III trial to demonstrate the efficacy of an antiangiogenic agent in pretreated patients with STS. However, these data could be further strengthened by identification of patients who are more likely to benefit from antiangiogenic agents such as pazopanib. Further studies are required to evaluate putative molecular and imaging markers of response to pazopanib and other antiangiogenic agents.

The decision to exclude all adipocytic STS was based on the outcome of the Phase II European Organisation for the Research and Treatment of Cancer study, where low progression-free survival was seen at 12 weeks in patients with liposarcoma. ${ }^{24}$ However, it is clear that liposarcoma is not one disease but rather three different biological diseases, ie, well differentiated/dedifferentiated, pleomorphic, and myxoid/round cell liposarcoma. A retrospective analysis of response to chemotherapy in patients with liposarcoma demonstrated a statistically significant higher response rate to first-line chemotherapy in patients with myxoid liposarcoma compared with well differentiated/dedifferentiated tumors (48\% versus $11 \%$, respectively). ${ }^{28}$ Theoretically, there may also be a differential response to pazopanib. A study of pazopanib (NCT01506596) is currently recruiting patients with dedifferentiated, myxoid/round-cell, pleomorphic, mixed-type, or not otherwise specified adipocytic sarcoma, which will yield results that could supplement the conclusions of the PALETTE trial.

Although a statistically significant improvement in progression-free survival was seen, there remains considerable room for improvement. There is hope that studies combining pazopanib with other agents may strengthen results. A randomized Phase II clinical trial using a combination of gemcitabine with or without pazopanib in treating patients with refractory STS is ongoing (ClinicalTrials.gov identifier: NCT01532687).

Very little is known about the activity of pazopanib in the neoadjuvant setting. Several clinical trials are exploring this option, either with preoperative pazopanib alone (NOPASS, ClinicalTrials.gov identifier NCT01543802) or in combination with doxorubicin and ifosfamide chemotherapy (ClinicalTrials.gov identifier NCT01446809).

\section{Mechanisms of resistance}

The success of pazopanib in clinical trials is tempered by the realization that for most patients the duration of disease stabilization is short-lived. Unfortunately, little is known about why certain STS subtypes, such as liposarcoma, may be resistant to pazopanib or how tumors sensitive to pazopanib acquire resistance during the course of treatment. This has been a problem with cancer therapy in general with agents inhibiting angiogenesis. Some patients will not benefit at all because some tumors appear to have an intrinsic pre-existing nonresponsiveness to antiangiogenic therapy. ${ }^{29,30}$ Some carcinomas are known to sometimes have increased proapoptotic signals from multiple different pathways. For example, in breast cancer, the presence of multiple proangiogenic factors is correlated negatively with prognosis. ${ }^{31}$ The presence of multiple non-VEGF factors has been correlated with in vitro resistance to VEGF inhibition in renal cell carcinoma and colon cancer cell lines. ${ }^{32}$

Even tumors with an excellent response to pazopanib will eventually develop resistance and it is unclear why this happens. Glioblastomas overcome cediranib-mediated VEGF receptor inhibition through increased production of fibroblast growth factor. ${ }^{33}$ Mice have been observed to upregulate production of VEGF rapidly following treatment 
with anti-VEGF receptor 2 antibodies. ${ }^{34}$ Increases in multiple proangiogenic factors have been observed following treatment with sunitinib. ${ }^{35}$

Increased activation of alternative proangiogenic signaling pathways, such as Delta-like 4-mediated Notch signaling, may further allow resistance to angiogenesis inhibitors. ${ }^{36}$ Inhibition of these pathways may results in excessive but nonproductive angiogenesis and thereby reduced tumor growth. ${ }^{37}$ The Tie receptors, together with their two major ligands, angiopoietin-1 and angiopoietin-2, are alternative pathways to induce critical features of angiogenesis, such as vessel maturation, ${ }^{38}$ and may be activated in cases of some resistance to angiogenesis inhibition.

Further, hypoxic conditions cause secretion of factors such as hypoxia-inducible factor- $1 \alpha$ that can have strong proangiogenic effects, including causing increased production of proangiogenic cytokines and growth factors. ${ }^{39}$ This can further lead to recruitment of endothelial and pericyte progenitor cells from the bone marrow which can form blood vessel components and recruit other factors necessary for angiogenesis ${ }^{40}$ or recruit tumor-derived macrophages which do not form blood vessels and may help recruit blood vessel formation. ${ }^{41}$ Pericytes may support blood vessel walls and decrease the necessity for proangiogenic factors, such as VEGF. 42,43

Also, phosphoinositide 3-kinase inhibitors may not only restrain tumor growth by inhibition of tumor angiogenesis, but can exert additional inhibitory effects on tumor cells. ${ }^{44}$ Sunitinib, for example, directly inhibits tumor cell proliferation and clonogenic capacity. ${ }^{45}$ Therefore, acquired resistance may also be a consequence of alternative signaling of tumor cells including the production of alternative angiogenic growth factors. Although tumors frequently overcome cytotoxic chemotherapy by acquisition of genetic mutations leading to decreased target binding or increased drug efflux, ${ }^{46}$ there is currently no evidence for such a mechanism leading to pazopanib resistance.

\section{Conclusion}

A randomized, placebo-controlled Phase III trial has shown that pazopanib has activity in advanced pretreated STS. The drug is well tolerated, allowing prolonged therapy. The drug has been approved for patients with sarcoma previously treated with chemotherapy. It is clear that angiogenesis has a critical role in STS, but further work is required to understand mechanisms of resistance and identify biomarkers of drug efficacy, as well as effectively and safely combining pazopanib with other agents targeting pathways that may be implicated in pazopanib resistance. Current and planned trials are evaluating pazopanib in combination with other agents as well as in the neoadjuvant setting.

\section{Disclosure}

The authors report no conflicts of interest in this work.

\section{References}

1. Casali PG, Blay JY. Soft tissue sarcomas: ESMO clinical practice guidelines for diagnosis, treatment and follow-up. Ann Oncol. 2010; 21 Suppl 5:198-203.

2. Penel N, Bui BN, Bay JO, et al. Phase II trial of weekly paclitaxel for unresectable angiosarcoma: the ANGIOTAX Study. J Clin Oncol. 2008;26:5269-5274

3. Le Cesne A, Blay JY, Judson I, et al. Phase II study of ET-743 in advanced soft tissue sarcomas: a European Organisation for the Research and Treatment of Cancer (EORTC) Soft Tissue and Bone Sarcoma Group trial. J Clin Oncol. 2005;3:576-584.

4. Maki RG, Wathen JK, Patel SR, et al. Randomized phase II study of gemcitabine and docetaxel compared with gemcitabine alone in patients with metastatic soft tissue sarcomas: results of sarcoma alliance for research through collaboration study 002 [corrected]. J Clin Oncol. 2007;25:2755-2763.

5. García-Del-Muro X, López-Pousa A, Maurel J, et al. Randomized phase II study comparing gemcitabine plus dacarbazine versus dacarbazine alone in patients with previously treated soft tissue sarcoma: a Spanish Group for Research on Sarcomas study. J Clin Oncol. 2011;29: 2528-2533.

6. Yoon SS, Segal NH, Olshen AB, Brennan MF, Singer S. Circulating angiogenic factor levels correlate with extent of disease and risk of recurrence in patients with soft tissue sarcoma. Ann Oncol. 2004;15: 1261-1266.

7. Rutkowski P, Kaminska J, Kowalska M, Ruka W, Steffen J. Cytokine serum levels in soft tissue sarcoma patients: correlations with clinicopathological features and prognosis. Int J Cancer Suppl. 2002;100: 463-471.

8. Graeven U, Andre N, Achilles E, Zornig C, Schmiegel W. Serum levels of vascular endothelial growth factor and basic fibroblast growth factor in patients with soft-tissue sarcoma. J Cancer Res Clin Oncol. 1999;125: 577-581.

9. Hayes AJ, Mostyn-Jones A, Koban MU, A'Hern R, Burton P, Thomas JM. Serum vascular endothelial growth factor as a tumour marker in soft tissue sarcoma. Br J Surg. 2004;91:242-247.

10. Yoon SS, Segal NH, Park PJ, et al. Angiogenic profile of soft tissue sarcomas based on analysis of circulating factors and microarray gene expression. J Surg Res. 2006;135:282-290.

11. Betsholtz C. Insight into the physiological functions of PDGF through genetic studies in mice. Cytokine Growth Factor Rev. 2004;15: 215-228.

12. Ferrara N. VEGF as a therapeutic target in cancer. Oncology. 2005;69 Suppl 3:11-16.

13. Bergers G, Song S, Meyer-Morse N, Bergsland E, Hanahan D. Benefits of targeting both pericytes and endothelial cells in the tumor vasculature with kinase inhibitors. J Clin Investig. 2003;111:1287-1295.

14. Erber R, Thurnher A, Katsen AD, et al. Combined inhibition of VEGF and PDGF signaling enforces tumor vessel regression by interfering with pericyte-mediated endothelial cell survival mechanisms. FASEB J. 2004;18:338-340.

15. George S, Merriam P, Maki RG, et al. Multicenter phase II trial of sunitinib in the treatment of nongastrointestinal stromal tumor sarcomas. J Clin Oncol. 2009;27:3154-3160.

16. Maki RG, D'Adamo DR, Keohan ML, et al. Phase II study of sorafenib in patients with metastatic or recurrent sarcomas. J Clin Oncol. 2009;27:3133-3140. 
17. Fox E, Aplenc R, Bagatell R, et al. A phase 1 trial and pharmacokinetic study of cediranib, an orally bioavailable pan-vascular endothelial growth factor receptor inhibitor, in children and adolescents with refractory solid tumors. J Clin Oncol. 2010;28:5174-5181.

18. Schwartz GK, Maki RG, Ratain MJ, et al. Brivanib (BMS-582664) in advanced soft-tissue sarcoma (STS): biomarker and subset results of a phase II randomized discontinuation trial. J Clin Oncol. 2011; 29 Suppl:Abstr 10000.

19. Harris PA, Boloor A, Cheung M, et al. Discovery of 5-[[4[(2,3-dimethyl-2H-indazol-6-yl)methylamino]-2-pyrimidinyl] amino]-2-methyl-benzenesulfonamide (pazopanib), a novel and potent vascular endothelial growth factor receptor inhibitor. $J$ Med Chem. 2008;51:4632-4640.

20. Bukowski RM. Pazopanib: a multikinase inhibitor with activity in advanced renal cell carcinoma. Expert Rev Anticancer Ther. 2010;10: 635-645.

21. Kumar R, Knick VB, Rudolph SK, et al. Pharmacokinetic-pharmacodynamic correlation from mouse to human with pazopanib, a multikinase angiogenesis inhibitor with potent antitumor and antiangiogenic activity. Mol Cancer Ther. 2007;6:2012-2021.

22. Hurwitz HI, Dowlati A, Saini S, et al. Phase I trial of pazopanib in patients with advanced cancer. Clin Cancer Res. 2009;15:4220-4227.

23. Keisner SV, Shah SR. Pazopanib: the newest tyrosine kinase inhibitor for the treatment of advanced or metastatic renal cell carcinoma. Drugs. 2011;71:443-454.

24. Sleijfer S, Ray-Coquard I, Papai Z, et al. Pazopanib, a multikinase angiogenesis inhibitor, in patients with relapsed or refractory advanced soft tissue sarcoma: a phase II study from the European Organisation for Research and Treatment of cancer-soft tissue and bone sarcoma group (EORTC study 62043). J Clin Oncol. 2009;27:3126-3132.

25. van der Graaf WT, Blay JY, Chawla SP, et al. Pazopanib for metastatic soft-tissue sarcoma (PALETTE): a randomised, double-blind, placebo-controlled phase 3 trial. Lancet. 2012;379:1879-1886.

26. Pàez-Ribes M, Allen E, Hudock J, et al. Antiangiogenic therapy elicits malignant progression of tumors to increased local invasion and distant metastasis. Cancer Cell. 2009;15:220-231.

27. Mackey JR, Kerbel RS, Gelmon KA, et al. Controlling angiogenesis in breast cancer: a systematic review of anti-angiogenic trials. Cancer Treat Rev. 2012;38:673-688.

28. Jones RL, Fisher C, Al-Muderis O, Judson IR. Differential sensitivity of liposarcoma subtypes to chemotherapy. Eur J Cancer. 2005;41: 2853-2860.

29. Broxterman HJ, Gotink KJ, Verheul HM. Understanding the causes of multidrug resistance in cancer: a comparison of doxorubicin and sunitinib. Drug Resist Updat. 2009;12:114-126.

30. Bergers G, Hanahan D. Modes of resistance to anti-angiogenic therapy. Nat Rev Cancer. 2008;8:592-603.

31. Relf M, LeJeune S, Scott PA, et al. Expression of the angiogenic factors vascular endothelial cell growth factor, acidic and basic fibroblast growth factor, tumor growth factor beta-1, platelet-derived endothelial cell growth factor, placenta growth factor, and pleiotrophin in human primary breast cancer and its relation to angiogenesis. Cancer Res. 1997;57:963-969.
32. Fernando NT, Koch M, Rothrock C, et al. Tumor escape from endogenous, extracellular matrix-associated angiogenesis inhibitors by up-regulation of multiple proangiogenic factors. Clin Cancer Res. 2008; 14:1529-1539.

33. Batchelor TT, Sorensen AG, di Tomaso E, et al. AZD2171, a pan-VEGF receptor tyrosine kinase inhibitor, normalizes tumor vasculature and alleviates edema in glioblastoma patients. Cancer Cell. 2007;11: 83-95.

34. Bocci G, Man S, Green SK, et al. Increased plasma vascular endothelial growth factor (VEGF) as a surrogate marker for optimal therapeutic dosing of VEGF receptor-2 monoclonal antibodies. Cancer Res. 2004;64:6616-6625.

35. Ebos JM, Lee CR, Christensen JG, Mutsaers AJ, Kerbel RS. Multiple circulating proangiogenic factors induced by sunitinib malate are tumor-independent and correlate with antitumor efficacy. Proc Natl Acad Sci U S A. 2007;104:17069-17074.

36. Ellis LM, Hicklin DJ. Pathways mediating resistance to vascular endothelial growth factor-targeted therapy. Clin Cancer Res. 2008;14: 6371-6375.

37. Thurston G, Noguera-Troise I, Yancopoulos GD. The Delta paradox: DLL4 blockade leads to more tumour vessels but less tumour growth. Nat Rev Cancer. 2007;7:327-331.

38. Augustin HG, Koh GY, Thurston G, Alitalo K. Control of vascular morphogenesis and homeostasis through the angiopoietin-Tie system. Nat Rev Mol Cell Biol. 2009;10:165-177.

39. Du R, Lu KV, Petritsch C, Liu P, et al. HIF1alpha induces the recruitment of bone marrow-derived vascular modulatory cells to regulate tumor angiogenesis and invasion. Cancer Cell. 2008;13:206-220.

40. Asahara T, Murohara T, Sullivan A, et al. Isolation of putative progenitor endothelial cells for angiogenesis. Science. 1997;275:964-967.

41. Pollard JW. Tumour-educated macrophages promote tumour progression and metastasis. Nat Rev Cancer. 2004;4:71-78.

42. Orlidge A, D'Amore PA. Inhibition of capillary endothelial cell growth by pericytes and smooth muscle cells. J Cell Biol. 1987;105: $1455-1462$.

43. Xian X, Håkansson J, Ståhlberg A, et al. Pericytes limit tumor cell metastasis. J Clin Investig. 2006;116:642-651.

44. Ellis LM, Hicklin DJ. VEGF-targeted therapy: mechanisms of anti-tumour activity. Nat Rev Cancer. 2008;8:579-591.

45. Gotink KJ, Rudek MA, Broxterman HJ, et al. The antiangiogenic tyrosine kinase inhibitor sunitinib has direct antitumor activity on renal and colorectal cancer cells in vitro at intratumoral concentrations achieved in mice. Abstract 150 presented at the 100th Annual Meeting of the American Association of Cancer Research, Denver, CO, April 18-22, 2009.

46. O'Connor R, Clynes M, Dowling P, O’Donovan N, O'Driscoll L. Drug resistance in cancer - searching for mechanisms, markers and therapeutic agents. Expert Opin Drug Metab Toxicol. 2007;3:805-817.
OncoTargets and Therapy

\section{Publish your work in this journal}

OncoTargets and Therapy is an international, peer-reviewed, open access journal focusing on the pathological basis of all cancers, potential targets for therapy and treatment protocols employed to improve the management of cancer patients. The journal also focuses on the impact of management programs and new therapeutic agents and protocols on

\section{Dovepress}

patient perspectives such as quality of life, adherence and satisfaction The manuscript management system is completely online and includes a very quick and fair peer-review system, which is all easy to use. Visit http://www.dovepress.com/testimonials.php to read real quotes from published authors. 